\title{
Ensuring performance requirements for semiactive suspension with nonconventional control systems via robust linear parameter varying framework
}

\author{
Balázs Németh $\odot$ | Péter Gáspár
}

Systems and Control Laboratory, Institute for Computer Science and Control, Budapest, Hungary

\section{Correspondence}

Balázs Németh, Systems and Control Laboratory, Institute for Computer Science and Control, H-1111 13-17., Kende utca, Budapest, Hungary.

Email: balazs.nemeth@sztaki.hu

\section{Funding information}

Magyar Tudományos Akadémia, Grant/Award Number: János Bolyai Research Scholarship; Ministry for Innovation and Technology, Hungary, Grant/Award Number: ÚNKP-20-5 New National Excellence Program; Nemzeti Kutatási Fejlesztési és Innovációs Hivatal, Grant/Award Number: NKFIH

2018-2.1.13-TÉT-FR

\begin{abstract}
Summary
In the article a method which is able to provide the required performance level of a system is proposed. Its principle is to combine the results of conventional control methods with those of methods based on nonconventional, for example, machine-learning-based ones. In more detail, it designs a robust linear parameter varying (LPV) control in a predefined form, whose output is equivalent to the output of a machine-learning-based control inside a predefined operational range. Outside of the operation range the output of the machine-learning-based control is overridden, while the intervention with the performance level is guaranteed. The efficiency of the proposed method is illustrated through an example on the semiactive suspension control design. The nonlinearities in the dynamics of the magneto-rheological damper are considered through a nonlinear parameter varying (NLPV) model. It designs an NLPV model-based LPV control, which is combined with a neural network to achieve preview capability.
\end{abstract}

\section{KE Y W O R D}

machine-learning-based control, performance guarantees, robust LPV control

\section{1 | INTRODUCTION AND MOTIVATION}

The increasing complexity of control design and decision making may result in combined applications of various control systems. One of the most important fields of this fusion is related to the control of autonomous vehicles, in which several driving features must be automated to reduce human interventions, for example, sensing the environment, making decisions, trajectory design, control and intervention with smart actuators. The complex architecture contains conventional control systems, for example, a model based optimal/robust solutions, and further nonconventional control systems, for example, learning-based methods, simultaneously.

Nevertheless, the increasing complexity of control systems poses the challenge of performance guarantees for the designers. In the design of conventional control systems the performances of the system can be defined in a mathematical form. Since there may be conflicts between the various performances, a balance between the levels of the performances must be found, for example, the application of weighting functions, iterative tuning, and so on. An advantage of conventional methods is that the yielded controller, in theory, guarantees the performance level of the closed-loop system. But, in case of complex systems the mathematical formulation of the performances may be difficult, especially in human-machine systems. For example, in the case of autonomous vehicles it can be difficult to describe formally 
the traveling comfort or the attributes of the human driving. These features have high importance in the design of the suspension control systems to achieve customer satisfaction. ${ }^{1}$

Nonconventional methods may provide a possible solution to the problem. For example, with imitation learning techniques it is not necessary to formulate the mathematical description of the system or the performances. The training set contains samples of the expected operation of the system, which meet human performance requirements. ${ }^{2,3}$ Another example is reinforcement learning, in which the performance requirements can be achieved through high number of learning scenarios in a training process. ${ }^{4,5}$ Although the different types of enhanced machine-learning methods are able to solve various control tasks effectively, the resulting performance level is not theoretically guaranteed, that is, the numerous training samples cannot guarantee the avoidance of the performance degradation in any scenario.

A challenge for the control design is to achieve the performance improvements of the nonconventional solutions, while the minimum performance level is guaranteed. It requires the combined application of conventional and learning-based control solutions. The difficulty in the analysis and control design of a combined system is the differences in the mathematical structure of machine-learning-based algorithms and formulation of the conventional dynamic controllers. However, there are some approaches on some control design problems in the existing literature. For example, Zhai et $\mathrm{l}^{6}$ propose the design for a class of discrete-time single-input single-output nonaffine uncertain nonlinear system. In the design the purpose of the linear dynamic controller is to stabilize the linearized system, while the state and output feedback adaptive neural network handles with nonlinearity. In Reference 7 a switching controller is developed, which consists of a traditional adaptive neural controller and an extra robust controller to pull back the transient from outside of the approximation domain. As a result of the method, the system output converges to a small neighborhood of the reference signal and the closed-loop system is globally stable. A repetitive learning approach based on predictive framework is presented in Reference 8 . The goal of the method is to construct recursively terminal set and terminal cost from state and input trajectories of previous iterations, while the feasibility and the nondecreasing property of the performances are guaranteed. The method incorporates in the learning feature, and thus, it is incompatible with the distinct machine-learning structures.

The contribution of the article is 2-fold, that is, it can be described from a theoretical viewpoint and from the side of its application. The theoretical contribution of the article is a novel control design framework based on the robust LPV theory, in which the conventional and nonconventional control components are combined. The design is based on an iteration procedure, whose result is an LPV controller, which guarantees the performance level of the combined system. The principle of the method is that a robust LPV control is designed, whose output signal is equivalent to the output signal of the nonconventional controller. Therefore, in the method the control input signal is expressed as a multiplication of the LPV controller output, a specified scheduling variable, together with a specified additive disturbance. By using the scheduling variable and the additive disturbance a wide range of the outputs can be covered. The advantage of the proposed method is that it is independent of the structure of the applied nonconventional control. Since the method uses only the input and output signals of the nonconventional control system, it can effectively be used for various control structures and agents such as machine-learning, intelligent PID, fuzzy logic controllers, heuristic approaches, and so on.

Due to the motivation of autonomous vehicles, especially the semiactive suspension systems, the design of LPV control with learning-based agents in the loop are in focus of this article. An important topic of semiactive suspension systems is the coordination of their intervention with the functionalities of the autonomous vehicles. For example, a performance of the automated velocity selection is to provide energy-efficient, safe and comfortable motion, see, for example, Reference 9. The energy, safety, and traveling comfort performance requirements in the vertical dynamics are partially guaranteed by the suspension control. Thus, the route and velocity selection might not be separated from the suspension control to achieve improved performances for the autonomous vehicle. There are various methods to consider forthcoming road information about the road condition in the suspension control design, for example, through a preview control ${ }^{10,11}$ or through road estimation. ${ }^{12}$ Nevertheless, an enhanced handling of the forthcoming road conditions may require various information sources and the handling of more dynamical motions, which may require nonconventional control solutions, for example, the forthcoming road section is assumed to be reached from LiDAR information, which can use deep-learning-based algorithms for the determination of road roughness. ${ }^{13-15}$

Although there are several solutions to the control design of semiactive suspension systems (eg, Skyhook, ${ }^{16} \mathcal{H}_{\infty}$, gain scheduling, ${ }^{1}$ and predictive methods ${ }^{12}$ ), the proposed control structure has the advantage of the capability of the preview and the possibility of using increased number of external signals. Thus, the contribution of the article from the side of the application is a semiactive suspension control design framework with which the minimization of the vertical acceleration is improved. It is achieved through the road information on the horizon ahead of the vehicle, which is processed 
by a neural network in the control loop. In the proposed design process the nonlinearities in the dynamics of the magnetorheological damper are formed through the nonlinear parameter varying (NLPV) method, and then, the NLPV model is transformed to be incorporated in a LPV-based control design. Thus, the resulting suspension control structure contains nonconventional and LPV-based controllers, whose roles differ in the intervention. The nonconventional controller is designed to maximize the performance level in the vertical acceleration of the system, that is, to achieve minimum vertical acceleration. However, the minimum performance level of the system with the nonconventional controller, that is, the maximum of the vertical acceleration is not guaranteed. The result of the iteration is a closed-loop system whose minimum performance level is guaranteed. In this sense the minimum performance level represents the worst case of the performance level, that is, in the suspension control example the upper bounds on the acceleration and on the compression are guaranteed.

The article is organized as follows. Section 2 proposes the concept of the method, the control rule and the structure of the control architecture. Section 3 proposes the selection of the values and the domains for the scheduling variable and the known disturbance, which are the fundamental elements of the control concept. The iterative design of the LPV control together with the optimization of the scheduling variable and disturbance domains are proposed in Section 4 . Then, the method is applied on the semiactive suspension control design problem as presented in Section 5. Finally, the contributions of the proposed method are summarized in Section 6.

\section{2 | DESIGN CONCEPT OF ROBUST LPV CONTROL}

The purpose of the concept is to form the structure of the LPV design, in which the output of the resulting controller is able to generate the output of the machine-learning-based control. The generation of the equivalent signal requires the appropriate selection of the scheduling variables and the structure of the known disturbance, which are important features of the proposed method.

The output of the machine-learning control is $u_{L} \in n$ is as follows

$$
u_{L}=\mathcal{F}\left(y_{L}\right)
$$

where $u_{L}=\left[\begin{array}{llll}u_{L, 1} & u_{L, 2} & \ldots & u_{L, n}\end{array}\right]^{T}, \quad y_{L} \in m_{L}$ contains the inputs of the controller and $\mathcal{F}$ represents the machine-learning-based controller. The output of a robust LPV control is $u_{K} \in n$ is as follows

$$
u_{K}=\mathcal{K}\left(\rho_{K}, y_{K}\right) \text {, }
$$

where $u_{K}=\left[\begin{array}{llll}u_{K, 1} & u_{K, 2} & \ldots & u_{K, n}\end{array}\right]^{T}, y_{K} \in m_{K}$ contains the measured signals and $\mathcal{K}$ represents the LPV controller with an $n$-element vector of scheduling variables $\rho_{K} \in \rho_{K}$.

The fundamental assumption of the design method is that the control input of the system $u=\left[\begin{array}{llll}u_{1} & u_{2} & \ldots & u_{n}\end{array}\right]^{T}$ can be expressed as a function of $u_{K}$ in a linear form by predefined conditions. The parameters in the linear formulation are selected to guarantee $u:=u_{L}$. Here the relationship among $u, u_{K}$ and $u_{L}$ is formed as follows

$$
u=I_{n \times n} \circ\left(\rho_{L}^{*} J_{1 \times n}\right) u_{K}+\Delta_{L}^{*}:=u_{L},
$$

where $\circ$ represents Hadamard product, $I_{n \times n}$ is an identity matrix, $J_{1 \times n}$ is a vector with one row, $\rho_{L}^{*}$ and $\Delta_{L}^{*}$ are vectors with $n$ elements as

$$
\begin{gathered}
\rho_{L}^{*}=\left[\begin{array}{llll}
\rho_{L, 1}^{*} & \rho_{L, 2}^{*} & \ldots & \rho_{L, n}^{*}
\end{array}\right]^{T}, \quad \rho_{L, i}^{*} \in \rho_{L, i}, \\
\Delta_{L}^{*}=\left[\begin{array}{llll}
\Delta_{L, 1}^{*} & \Delta_{L, 2}^{*} & \ldots & \Delta_{L, n}^{*}
\end{array}\right]^{T}, \quad \Delta_{L, i}^{*} \in \Lambda_{L, i},
\end{gathered}
$$

and $\rho_{L, i}^{*}, \Delta_{L, i}^{*}$ are time-dependent weighting signals. $\varrho_{L, i}=\left[\rho_{L, i, \min } ; \rho_{L, i, \max }\right], \Lambda_{L, i}=\left[\Delta_{L, i, \min } ; \Delta_{L, i, \max }\right]$ represent domains in (3), where $\rho_{L, i, \min }, \rho_{L, i, \max }, \Delta_{L, i, \min }, \Delta_{L, i, \max }$ are scalars. The sets of the domains are denoted by $\rho_{L}, \Lambda_{L}$. Since $I_{n \times n} \circ\left(\rho_{L}^{*} J_{1 \times n}\right)$ leads to a diagonal matrix with the related elements of $\rho_{L}^{*}$, the signal $u_{i}$ depends only on $u_{K, i}$. If the conditions (3) for $\rho_{L, i}^{*}$ and $\Delta_{L, i}^{*}$ are guaranteed, the control input of the system $u$ is equal to $u_{L}$. But, if there exists at least one $i \in[1 ; n]$, where 


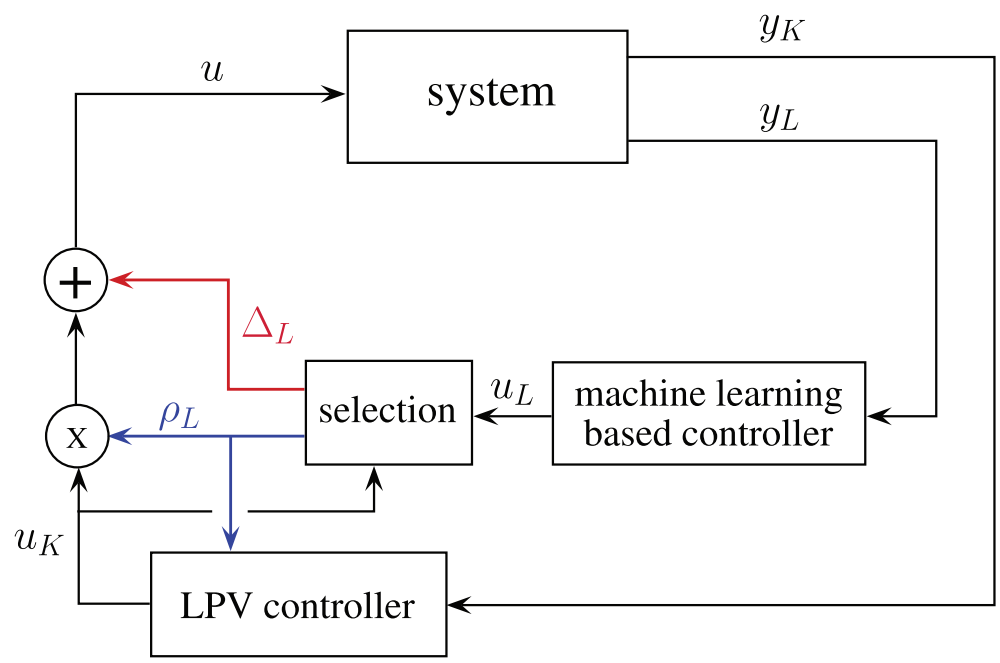

F I G U R E 1 Structure of the control architecture

$\rho_{L, i}^{*} \notin \varrho_{L, i}$ or $\Delta_{L, i}^{*} \notin \Lambda_{L, i}$, the variables $\rho_{L, i}^{*}, \Delta_{L, i}^{*}$ are limited with the boundaries of $\rho_{L, j}$ and $\Lambda_{L, j}$ during the computation of the control signal $u_{i}$. In this case $u \neq u_{L}$.

The general control rule, which contains both cases is formed as

$$
u=I_{n \times n} \circ\left(\rho_{L} J_{1 \times n}\right) u_{K}+\Delta_{L}
$$

where

$$
\begin{aligned}
& \rho_{L}=\left[\begin{array}{llll}
\rho_{L, 1} & \rho_{L, 2} & \ldots & \rho_{L, n}
\end{array}\right]^{T}, \\
& \Delta_{L}=\left[\begin{array}{llll}
\Delta_{L, 1} & \Delta_{L, 2} & \ldots & \Delta_{L, n}
\end{array}\right]^{T}, \\
& \rho_{L, i}=\min \left(\max \left(\stackrel{*}{\rho} ; \rho_{L, i}, i, \max \right) ; \rho_{L, i, \min }\right), \quad \forall i=1 \ldots n, \\
& \Delta_{L, i}=\min \left(\max \left(\underset{L, i}{*} ; \Delta_{L, i, \min }\right) ; \Delta_{L, i, \max }\right), \quad \forall i=1 \ldots n .
\end{aligned}
$$

The relations (6c)-(6d) guarantee that $\rho_{L} \in \varrho_{L}$ and $\Delta_{L} \in \Lambda_{L}$. The relationship among $u_{L}, u_{K}$, $u$ and the structure of the control architecture is illustrated in Figure 1. In the proposed concept the feedback loop contains the LPV controller, while the machine-learning-based controller is in an auxiliary loop. The role of the selection block in the architecture is to select $\rho_{L}, \Delta_{L}$. The actuated control input $u$ on the system depends on $u_{K}, \rho_{L}, \Delta_{L}$.

The architecture shows the main idea of the proposed concept. The minimum performance level is determined by the LPV controller in the entire operation domain of the system, while inside of the domains $\varrho_{L}, \Lambda_{L}$ the performance level is enhanced through machine-learning-based control. Thus, the advantages of machine-learning-based control can be achieved, while its drawback, such as performance degradation in some scenarios is eliminated through the guaranteed minimum performance level.

The design of the control architecture requires the following steps in the process:

1. It is necessary to select the values of $\rho_{L}, \Delta_{L}$ and the domains $\rho_{L}, \Lambda_{L}$, as defined in (5) and (6).

2. The robust LPV control must be designed, in which the domains $\varrho_{L}, \Lambda_{L}$ are incorporated.

The challenge of the control design is that the determination of $\varrho_{L}, \Lambda_{L}$ and the LPV design are not independent of each other. The control design requires the selection of the domains, while the effective selection of the domains requires experience on the performance of the design control. As a solution to this anomaly, an iterative design method is proposed, which incorporates the domain selection and the LPV design in itself. The proposed approach is focused on the iterative design, in which the machine-learning-based control is considered to be available. 


\section{3 | SELECTION OF THE VALUES AND DOMAINS FOR SCHEDULING VARIABLES AND MEASURED DISTURBANCE}

In this section two strategies are proposed. First, the current values of $\rho_{L}$ and $\Delta$ based on the control signal vectors $u_{L}, u_{K}$ are calculated. Second, a method for the selection of the domains $\varrho_{L}, \Lambda_{L}$ is proposed.

\subsection{Calculation of the scheduling variable and the measured disturbance}

The selection strategy of $\rho_{L}$ and $\Delta$ is based on the relation between $u_{L}$ and $u_{K}$, see (5). Due to the expression $I_{n \times n} \circ\left(\rho_{L}^{*} J_{1 \times n}\right) u_{K}$ in (3) the control input $u_{i}$ is independent of $u_{K, j}$, for all $i \neq j ; i, j \in[i ; n]$, as it is detailed in Section 2 . Thus, the selection of the pairs $\rho_{L, i}^{*}, \Delta_{L, i}^{*}$ is also independent of $\rho_{L, j}^{*}, \Delta_{L, j}^{*}$ for all $i \neq j ; i, j \in[i ; n]$. As a result, the control signals of the system with multiple control input can be independently examined.

In relation (3) $u_{i}:=u_{L, i}$, which means that

$$
u_{L, i}=\rho_{L, i}^{*} u_{K, i}+\Delta_{L, i}^{*}, \quad \forall i=1 \ldots n
$$

in which $\rho_{L, i}^{*} \in \varrho_{L, i}$ and $\Delta_{L, i}^{*} \in \Lambda_{L, i}$. The presented formulation of $u_{L, i}$ shows that if $u_{K, i}$ is close to $u_{L, i}$, then relation (7) can be effectively guaranteed through $\rho_{L, i}^{*}$. But, if $\left|u_{K, i}\right|<\epsilon$, where $\epsilon>0$ has a small value, then $\rho_{L, i}^{*} u_{K, i}$ has a low impact on $u_{L, i}$, see (7). In this case $\Delta_{L, i}^{*}$ must be selected close to $u_{L, i}$. Due to these specialties of the formulation (7), a selection method of $\rho_{L, i}^{*}, \Delta_{L, i}^{*}$ has been developed, which contains two distinguished scenarios, depending on the relation between $u_{L, i}$ and $u_{K, i}$.

In the first case the relationship between $u_{L, i}$ and $u_{K, i}$ can be expressed as

$$
\begin{gathered}
\left|u_{L, i}\right| \geq \rho_{L, i, \min }\left|u_{K, i}\right|, \quad \text { and } \\
\operatorname{sgn}\left(u_{L, i}\right)=\operatorname{sgn}\left(u_{K, i}\right), \quad \text { and } \\
u_{K, i} \neq 0,
\end{gathered}
$$

where $\rho_{L, i, m i n}>0$ is the lower bound of the domain $\varrho_{L, i}$. It means that in this scenario $u_{L, i}$ and $u_{K, i}$ have the same signs, while $\left|u_{L, i}\right|$ has a high value. Thus, if conditions (8) are guaranteed, the selection method of $\rho_{L, i}^{*}, \Delta_{L, i}^{*}$ is

$$
\begin{gathered}
\rho_{L, i}^{*}=\frac{u_{L, i}}{u_{K, i}}, \\
\Delta_{L, i}^{*}=0 .
\end{gathered}
$$

Examples of this scenario in the unshaded parts of Figure 2 are illustrated.

The second case contains the rest of the possible relationship between $u_{L, i}$ and $u_{K, i}$, which is expressed as

$$
\begin{gathered}
\left|u_{L, i}\right|<\rho_{L, i, \min }\left|u_{K, i}\right|, \quad \text { and } \\
\operatorname{sgn}\left(u_{L, i}\right) \neq \operatorname{sgn}\left(u_{K, i}\right), \quad \text { and } \\
u_{K, i}=0 .
\end{gathered}
$$

In this case the measured disturbance $\Delta_{L, i}$ has important role, for example, it is able to handle the zero transitions of the signals. In this case $\rho_{L, i}^{*}$ is fixed as $\rho_{L, i, m i n}$, while the difference between the signals is compensated for both $\rho_{L, i}^{*}$ and $\Delta_{L, i}^{*}$, such as

$$
\begin{gathered}
\rho_{L, i}^{*}=\rho_{L, i, \min }, \\
\Delta_{L, i}^{*}=u_{L, i}-\rho_{L, i, \min } u_{K, i} .
\end{gathered}
$$




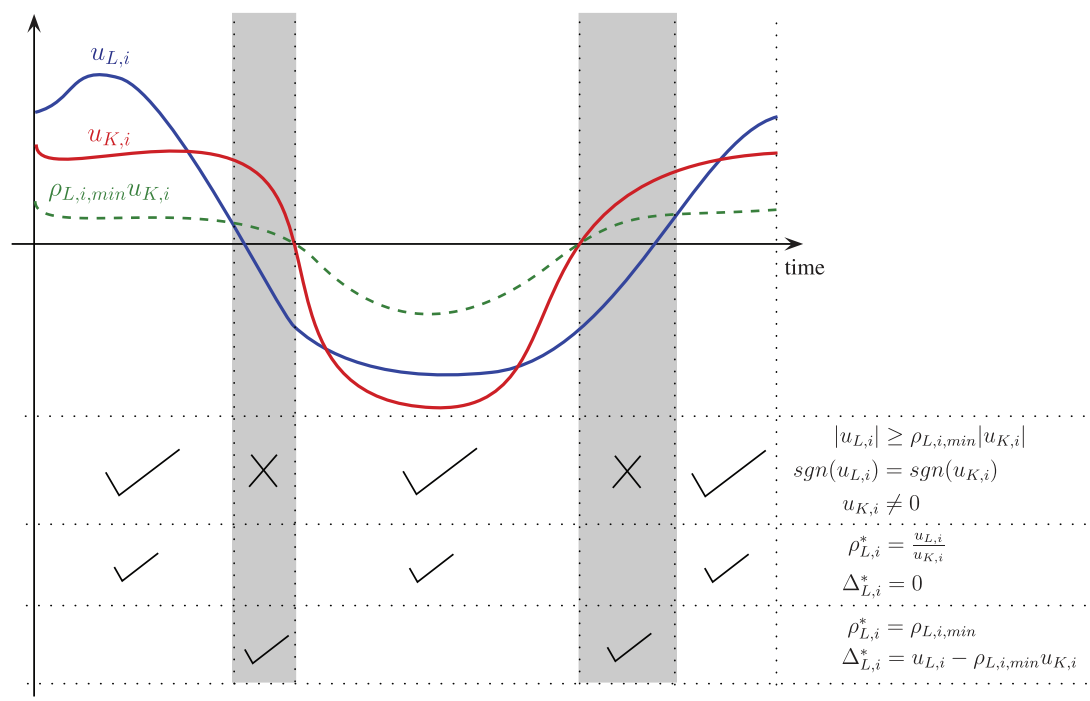

F I G U R E 2 Example on the selection of $\rho_{L, i}$ and $\Delta_{L, i}$

Figure 2 presents examples of the second scenario, in which the conditions (8) are not guaranteed, see the shaded parts of the illustration. The calculated $\rho_{L, i}^{*}, \Delta_{L, i}^{*}$ are used to provide the signals $\rho_{L, i}, \Delta_{L, i}$ based on the expressions (6c)-(6d).

\section{2 | Domains selection for the scheduling variable and the measured disturbance}

The generation of the control input $u$ requires not only the current values of $\rho_{L}$ and $\Delta_{L}$, but also their domains $\rho_{L}, \Lambda_{L}$, see (6). Similarly to the selection of $\rho_{L}, \Delta_{L}$, the related domain pairs $\varrho_{L, i}$ and $\Lambda_{L, i}$ can be selected independently of each other for all $i$. The boundaries of the domains are defined by the values $\rho_{L, i}=\left[\rho_{L, i, \min } ; \rho_{L, i, \max }\right]$ and $\Lambda_{L, i}=\left[\Delta_{L, i, \min } ; \Delta_{L, i, \max }\right]$, see Section 2 . The selection of the boundaries is based on scenarios, which can be yielded by simulations or experiments. In the following a selection method of all boundary values $\rho_{L, i, \min }, \rho_{L, i, \max }, \Delta_{L, i, \min }, \Delta_{L, i, \max }$ is proposed.

1. The upper bound of $\varrho_{L, i}$ is calculated based on the scenarios, where the conditions (8) are guaranteed. Using (9a) $\rho_{L, i, \max }$ is:

$$
\rho_{L, i, \max }=\max \left(\frac{u_{L, i}}{u_{K, i}}\right)
$$

2. The lower bound $\rho_{L, i, \min }>0$ has relevance in the scenarios, in which the conditions (8) are not satisfied. Its selection has impact on the domain $\Lambda_{L, i}$ as it is detailed below.

3. The upper bound of $\Lambda_{L, i}$ is determined based on the scenarios, in which the conditions (8) are not guaranteed. Its determination is based on relation (11b). Moreover, $\Delta_{L, i, \max }$ is reached, when $u_{L, i}>0$ and $u_{K, i}<0$, such as

$$
\Delta_{L, i, \max }=\max \left(u_{L, i}-\rho_{L, i, \min } u_{K, i}\right) .
$$

Relation (13) presents that the selection of $\rho_{L, i, \min }$ influences the value of the boundary $\Delta_{L, i, \max }$.

4. Similarly, the calculation of $\Delta_{L, i, m i n}$ is also based on (11b), when $u_{L, i}<0$ and $u_{K, i}>0$ :

$$
\Delta_{L, i, \min }=\min \left(u_{L, i}-\rho_{L, i, \min } u_{K, i}\right),
$$

which presents that $\rho_{L, i, m i n}$ has role in the lower bound of $\Lambda_{L, i}$ as well.

Relations (12)-(14) show that the selection of $\rho_{L, i, \min }$ has impact on both domains, that is, $\varrho_{L, i}$ and $\Lambda_{L, i}$. Moreover, the scenario-based computation of the boundaries induces that there are a trade-off between the range of the domains and the operation of the controllers. For example, if the boundaries of the domains $\varrho_{L, i}$ and $\Lambda_{L, i}$ are selected to have small ranges, then $\rho_{L, i}, \Delta_{L, i}$ can be slightly varied and the boundaries of their domains are often reached. Thus, $u_{i}$ often differs 
from $u_{L, i}$, which means that the advantages of the machine-learning-based controller are not exploited. In the other case, if the domains $\varrho_{L, i}, \Lambda_{L, i}$ are selected to have high ranges, $u_{i}$ is equal to $u_{L, i}$ in most of the interventions. However, if $u_{L, i}$ leads to a performance degradation, then it is not immediately avoided. This example presents that there is a trade-off between the range of the domains and the characteristics of the control intervention. In the following section the design of the LPV control is proposed, together with the selection of $\varrho_{L}, \lambda_{L}$. The two processes are set in an iterative framework.

\section{4 | ITERATIVE DESIGN OF THE LPV CONTROL}

The representation of the system is formed in the following control-oriented LPV state-space representation with $p \in N^{+}$ number of states as

$$
\dot{x}=A(\rho) x+B_{1}(\rho) w+B_{2}(\rho) u
$$

where $x$ represents a $p$-element state vector, $w$ contains the disturbances and $u=\left[\begin{array}{llll}u_{1} & u_{2} & \ldots & u_{n}\end{array}\right]^{T}$ vector incorporates the control inputs. $A(\rho), B_{1}(\rho), B_{2}(\rho)$ are matrices in the system representation, $\rho \in \rho$ vector contains the scheduling variables. In the following, the representation of the system (15) is used for the design of the robust LPV control $\mathcal{K}\left(\rho_{K}, y_{K}\right)$, see (2).

The purpose of the design is to derive the LPV controller which guarantees a minimum performance level for the closed-loop system, considering the predefined control rule (5). The output of the LPV controller $u_{K}$ is used in the expression $u=I_{n \times n} \circ\left(\rho_{L} J_{1 \times n}\right) u_{K}+\Delta_{L}$. Therefore, the state-space representation of the system (15) is reformulated through the relationship between $u$ and $u_{K}$ as

$$
\dot{x}=A\left(\rho_{K}\right) x+B_{1}\left(\rho_{K}\right) w_{K}+B_{2}\left(\rho_{K}\right) u_{K},
$$

where the vector of the scheduling variables $\rho_{K} \in \rho_{K}$ is composed as $\rho_{K}=\left[\begin{array}{ll}\rho & \rho_{L}\end{array}\right]^{T}, \varrho_{K}=\left[\begin{array}{ll}\rho & \rho_{L}\end{array}\right]^{T}$. The disturbance vector $w_{K}$ of the state-space representation (16) is composed as $w_{K}=\left[\begin{array}{ll}w & \Delta_{L}\end{array}\right]^{T}$ and the matrices are

$$
\begin{gathered}
A\left(\rho_{K}\right)=A(\rho), \\
B_{1}\left(\rho_{K}\right)=\left[\begin{array}{ll}
B_{1}(\rho) & B_{2}(\rho)
\end{array}\right], \\
B_{2}\left(\rho_{K}\right)=B_{2}(\rho)\left(I_{n \times n} \circ\left(\rho_{L} J_{1 \times n}\right)\right),
\end{gathered}
$$

where $\rho_{K}$ incorporates in $\rho$.

In the robust LPV framework the role of the controller is to guarantee a minimum performance level. ${ }^{17}$ Performance $z_{K}$ of the closed-loop system with $\mathcal{K}\left(\rho_{K}, y_{K}\right)$ is expressed through the control inputs $u$ and the existing disturbances $w$ as

$$
z_{K}=C_{2}(\rho) x+D_{21}(\rho) w+D_{22}(\rho) u .
$$

Similarly to the state-space representation (15)-(16), the performance equation (18) through $u=I_{n \times n} \circ\left(\rho_{L} J_{1 \times n}\right) u_{K}+\Delta_{L}$ is also reformulated as

$$
z_{K}=C_{2}\left(\rho_{K}\right) x+D_{21}\left(\rho_{K}\right) w_{K}+D_{22}\left(\rho_{K}\right) u_{K},
$$

where the matrices are

$$
\begin{gathered}
C_{2}\left(\rho_{K}\right)=C_{2}(\rho) x, \\
D_{21}\left(\rho_{K}\right)=\left[\begin{array}{ll}
D_{21}(\rho) & D_{22}(\rho)
\end{array}\right], \\
D_{22}\left(\rho_{K}\right)=D_{22}(\rho)\left(I_{n \times n} \circ\left(\rho_{L} J_{1 \times n}\right)\right),
\end{gathered}
$$

where $q \in \mathbf{N}^{+}$represents the number of performances in $z_{K}$. 
Finally, the input vector $y_{K}$ of the LPV controller $\mathcal{K}\left(\rho_{K}, y_{K}\right)$ must be expressed in the function of $x$, $w$, and $u$ for the design process. It is represented by the measurement equation, which has the form

$$
y_{K}=C_{1}(\rho) x+D_{11}(\rho) w+D_{12}(\rho) u .
$$

Through the relation $u=I_{n \times n} \circ\left(\rho_{L} J_{1 \times n}\right) u_{K}+\Delta_{L}$ the measurement equation is formed as

$$
y_{K}=C_{1}\left(\rho_{K}\right) x+D_{11}\left(\rho_{K}\right) w_{K}+D_{12}\left(\rho_{K}\right) u_{K},
$$

where the matrices of (22) are

$$
\begin{gathered}
C_{1}\left(\rho_{K}\right)=C_{1}(\rho) x+0_{m_{K} \times p} \rho_{L}, \\
D_{11}\left(\rho_{K}\right)=\left[\begin{array}{ll}
D_{11}(\rho) & \left.D_{12}(\rho)\right]+0_{m_{K} \times 2 n} \rho_{L}, \\
D_{12}\left(\rho_{K}\right)=D_{12}(\rho)\left(I_{n \times n} \circ\left(\rho_{L} J_{1 \times n}\right)\right),
\end{array}\right.
\end{gathered}
$$

where $0_{m_{K} \times p}, 0_{m_{K} \times 2 n}$ are zero matrices.

The result of the LPV control design method is that the closed-loop system is quadratically stable and the induced $\mathcal{L}_{2}$ norm from the extended disturbance vector $w_{K}$ to $z_{K}$ is less than the scalar $\gamma>0$. The existence of a controller that solves the quadratic LPV $\gamma$-performance problem can be expressed as the feasibility of a set of LMIs, which can be solved numerically. The constraints set by the LMIs are not finite. The infiniteness of the constraints is relieved by a finite, sufficiently fine grid. To specify the grid of the performance weights for the LPV design the scheduling variables are defined through lookup-tables. Gridding reflects the qualitative changes in the performance weights, that is, the scheduling variables $\rho_{K} \in \rho_{K}$. The stability and the performance level of the closed-loop system are guaranteed by the design procedure. ${ }^{17-19}$ The quadratic LPV performance problem is to choose the parameter varying controller $\mathcal{K}\left(\rho_{K}, y_{K}\right)$ in such a way that the resulting closed-loop system is quadratically stable and the induced $\mathcal{L}_{2}$ norm from the disturbance and the performances is less than the value $\gamma$. The minimization task is the following:

$$
\begin{gathered}
\inf _{\mathcal{K}\left(\rho_{K}, y_{K}\right)} \sup _{\rho_{K} \in \rho_{K}} \sup _{\left\|w_{K}\right\|_{2} \neq 0,} \frac{\left\|z_{K}\right\|_{2}}{\left\|w_{K}\right\|_{2}} . \\
w_{K} \in \mathcal{L}_{2}
\end{gathered}
$$

The existence of a controller that solves the quadratic LPV $\gamma$-performance problem can be expressed as the feasibility of a set of LMIs, which can be solved numerically. Finally, the state-space representation of the LPV control $\mathcal{K}\left(\rho_{K}, y_{K}\right)$ is constructed, ${ }^{17,20}$ which leads to the control input $u_{K}$, see (2). The input signal $u_{K}$ is incorporated in the computation of $u$ (see (5)) together with the selection of $\rho_{L}, \Delta_{L}$ (see Section 3). The control rule results in that the minimum performance level of the closed-loop system is determined by $\mathcal{K}\left(\rho_{K}, y_{K}\right)$. The details of control design process, that is, the LMI formulation, the selection of the grid and the weighting functions can be found in Reference 21 .

The relationship between the selection of $\rho_{L}, \Lambda_{L}$ and the design of $\mathcal{K}\left(\rho_{K}, y_{K}\right)$ has been presented in Section 3. The design problem of the robust LPV controller also forms interconnection, because the optimization task (24) is incorporated in the domains $\varrho_{L}, \Lambda_{L}$ through $\varrho$ and $w_{K}$. However, the determination of $\varrho_{L}, \Lambda_{L}$ requires preliminary information based on scenarios, which leads to an iterative design process.

The purpose of the iteration is to fit the domains to the intervention of the control signals $u_{K}, u_{L}$. The result of the fitting is the reduction of the domains and the reduction of the conservativeness of controller $\mathcal{K}\left(\rho_{K}, y_{K}\right)$. The fitting of the domains is achieved through an iterative process. Through the iteration a balance between the range of the domains and the characteristics of the control intervention can be achieved. Thus, in the iteration the domains and the characteristics of the intervention are incorporated, which leads to the following optimization task

$$
\min _{\substack{\rho_{L, i, \min }>0, \rho_{L, i, \max }>0}} \sum_{i=1}^{n} R_{i}\left(\rho_{L, i, \max }-\rho_{L, i, \min }\right)+D_{i}\left(\left|\Delta_{L, i, \max }\right|-\left|\Delta_{L, i, \min }\right|\right)+T_{i} \bar{E}_{i},
$$


with the constraint $\rho_{L, i, \max }>\rho_{L, i, \min }$, where $\bar{E}_{i}$ is the average relative error of $u_{i}$ and $u_{L, i}$. Moreover, $R_{i}>0, D_{i}>0$ and $T_{i}>0$, $i=1 \ldots n$ scalars are design parameters.

Through the selection of $T$ the average relative error of $u_{i}$ and $u_{L, i}$ can be scaled. The motivation behind the increasing of $T$ is to design an LPV controller whose output is as close as possible to the output of the nonconventional controller. If $u_{i}$ is close to $u_{L, i}$, the advantageous dynamics of the $\mathrm{NN}$ controller is approximated by the LPV controller. Its consequence is that the domains of $\varrho_{L, i}$ and $\Lambda_{L, i}$ are increased. Nevertheless, in a scenario with the performance loss of the nonconventional controller, $u_{i}$ can be close to $u_{L, i}$ for a long time, which can reduce the minimum performance level of the system. It motivates the limitation of $T$. The roles of $R_{i}, D_{i}$ parameters are to scale the domains and to guarantee a balance between them. Thus, the values of $R_{i}, D_{i}$ express priority between the reduction of the scheduling variable domain and the disturbance domain. The motivation behind the selection of $R, D$ is to facilitate the LPV control design. If the domain of $\varrho_{L, i}$ is increased, the grid of the LPV design is also increased. Due to the increased difference between the edges of the grid, the design process of the LPV controller can be difficult, because the domains of the systems with the frozen scheduling variables are high. Similarly, if the domain of $\Lambda_{L, i}$ is increased, an increased robustness against the system is required. Both effects can result in unfeasible LMI problems in the design of the LPV control. Therefore, it is necessary to limit $R$ and $D$.

The solution of the optimization problem (25) begins with domains with high ranges, which are reduced through the following iteration process:

1. The domains $\varrho_{L, i}=\left[\rho_{L, i, \min } ; \rho_{L, i, \max }\right]$ and $\Lambda_{L, i}=\left[\Delta_{L, i, \min } ; \Delta_{L, i, \max }\right]$ are selected high in the first step. The initial value of $\rho_{L, i, \min }$ is selected for $\rho_{L, i, \min }=\varepsilon$, where $0<\varepsilon$ has a small value. Initially, $\rho_{L, i, \max }$ is selected high and similarly, $\left|\Delta_{L, i, \min }\right|$, $\left|\Delta_{L, i, \max }\right|$ also have high values. This results in a conservative LPV control and the purpose of the iterative design process is to reduce the conservativeness through the appropriate selection of the boundaries.

2. The LPV control with the selected domains is designed using (24).

3. The closed-loop system with the incorporation of the designed $\mathcal{K}\left(\rho_{K}, y_{K}\right)$ and the domains $\varrho_{L}, \Lambda_{L}$ are analyzed through various scenarios. It yields in the signals $u_{L}$ and $u_{K}$.

4. Due to the results of the scenarios the boundaries are modified to reduce the cost function of the optimization problem (25). The new values of $\rho_{L, i, \max }$ for all $i=1 \ldots n$ are selected through (12), based on the scenarios. The values of $\rho_{L, i, m i n}$, $i=1 \ldots n$ are also modified, which have impacts on $\Delta_{L, i, \min }, \Delta_{L, i, \max }, i=1 \ldots n$, see (13) and (14).

5. The LPV design, the scenarios and the evaluation (steps 2-4) are performed until the minimum of (25) is reached. If the minimum performance level of the designed control is not suitable, or the ranges of the domains result in frequent control intervention on the bounds, the parameters $R_{i}, D_{i}$, and $T_{i}$ must be modified (step 1 ) and the iteration must be performed again.

The evaluation of the cost function in (25) and the setting of the optimization variables can be performed through, for example, simplex search or trust-region-reflective methods, see References 22,23.

\section{5 | ILLUSTRATION OF THE ITERATIVE DESIGN ON A SEMIACTIVE SUSPENSION CONTROL PROBLEM}

In this section the iterative LPV design method is illustrated on the example of a semiactive suspension control problem. The purpose of the control is to guarantee the comfortable vertical motion of the vehicle, while the road holding is also guaranteed. In the example the role of the machine-learning-based control is to provide a control input $u_{L}$, whose computation is partially based on the preview information about the road surface in a predicted horizon. Through the forthcoming road information the semiactive suspension is set to provide a comfortable traveling.

\section{1 | Design of the control system}

The training of the neural network is based on the scenarios, with which the weights of each neurons are set to achieve the minimum cumulated $\left|\ddot{z}_{s}\right|$. The inputs of the neural network are the current measured $\ddot{z}_{s}$ and the forthcoming vertical values of road profile $z_{r}$ on the horizon ahead of the vehicle. The output of the neural network is $u_{L}$. In the example the road prediction in 0.5 second horizon with the endpoints of three equidistant segments is considered. The training is performed through the Nelder-Mead simplex algorithm. ${ }^{22}$ 
The design of the LPV control is based on the vertical quarter-car model, which is extended with the dynamics of the semiactive damper: ${ }^{24}$

$$
\begin{gathered}
m_{s} \ddot{z}_{s}=-F_{s}-F_{d}, \\
m_{u s} \ddot{z}_{u s}=F_{s}+F_{d}-F_{t},
\end{gathered}
$$

where $m_{s}, m_{u s}$ and $z_{s}, z_{u s}$ are the sprung unsprung mass and their vertical motion, $F_{s}$ is the spring force and $F_{t}$ is the vertical tire force. $F_{d}$ contains the passive damping force of the damper and the active damping force $F_{e r}$, which is achieved by the electrical field in the damper. In this article $F_{d}$ is formulated based on Guo's model, see Reference 25 . The system can be transformed to a state-space representation in a NLPV form, such as

$$
\dot{x}=A x+B_{1} w+B_{2} \Phi(x) u,
$$

where $A, B_{1}, B_{2}$ are state matrices and the system is nonlinear in $x$. The state vector contains $x^{T}=$ $\left[\begin{array}{lllll}z_{s}-z_{u s} & \dot{z}_{s} & z_{u s}-z_{r} & \dot{z}_{u s} & F_{e r}\end{array}\right]$ and $w=\dot{z}_{r}$ contains the road profile derivative and $u \in[0 ; 1]$ control input is the duty cycle of PWM channel. Moreover, the hysteresis in the dynamics of the damper is represented by $\Phi(x)=\tanh (\Gamma x)$, where $\Gamma$ vector contains the hysteresis coefficients of the damper.

Since $u$ is a bounded signal, the input constraint in the control design must be considered. It is achieved through the division of the control input as $u=u_{s t}+u_{d y n}$, where $u_{s t}=0.5$ is a static control input. Moreover, $u_{d y n} \in[-0.5 ; 0.5]$ is a dynamic control input, which is computed by the controller. Since $u_{d y n}$ is centered around 0 , its limitation in the control design can be formulated through weighting function on it. The weighting function on $u_{d y n}$ must guarantee that $u_{d y n}$ is between $[-0.5 ; 0.5]$, with which the input constraint on $u$ is achieved. $u_{s t}$ is considered as a constant additional input disturbance in the design process.

The proposed control design problem on the NLPV system model is performed through the transformation of the NLPV model into an LPV model. It is achieved through hiding $\Phi(x)$ in a scheduling variable. Thus, the selection of $\rho=\Phi(x) \in[-1 ; 1]$ as a bounded scheduling variable of the system yields a LPV form of the system. Moreover, due to relation (6) another scheduling variable $\rho_{L}$ is introduced and $\rho_{K}=\left[\begin{array}{ll}\rho & \rho_{L}\end{array}\right]^{T}$ vector is formed. Thus, the resulted LPV representation of the system is

$$
\dot{x}=A x+B_{1} w_{K}+B_{2}\left(\rho_{K}\right) u_{K},
$$

in which $B_{2}\left(\rho_{K}\right)=B_{2} \rho \rho_{L}$ and $w_{K}=\left[\begin{array}{lll}w & \Delta_{L} & u_{s t}\end{array}\right]^{T}$.

The performances of the system are the minimization of the vertical acceleration of the sprung mass, the compression in the suspension and the control intervention $u_{d y n}$, which are formed as

$$
\begin{gathered}
z_{1}=\ddot{z}_{s}, \quad\left|z_{1}\right| \rightarrow \min , \\
z_{2}=z_{s}-z_{u s}, \quad\left|z_{2}\right| \rightarrow \min , \\
z_{3}=u_{d y n}=\rho_{L} u_{K}+\Delta_{L}, \quad\left|z_{3}\right| \rightarrow \text { min. }
\end{gathered}
$$

The performance vector $z_{K}$, which incorporates in the elements of (29) is expressed as

$$
z_{K}=C_{2} x+D_{21} w_{K}+D_{22}\left(\rho_{K}\right) u_{K}
$$

where $C_{2}, D_{21}$ and $D_{22}\left(\rho_{K}\right)=\left[\begin{array}{lll}0 & 0 & \rho_{L}\end{array}\right]^{T}$ are matrices. The controller has one measured signal, such as $\ddot{z}_{s}$, which can be expressed in the function of $x$ :

$$
y_{K}=C_{1} x,
$$

where $C_{1}$ is a vector. The yielded LPV system, which contains the system dynamics (28), the performances (30) and the measurement (31) is formed as

$$
\dot{x}=A x+B_{1} w_{K}+B_{2}\left(\rho_{K}\right) u_{K},
$$


T A B L E 1 Results of the optimization through the iterative control design

\begin{tabular}{llllllll}
$\boldsymbol{R}$ & $\boldsymbol{D}$ & $\boldsymbol{T}$ & $\rho_{L, \min }$ & $\rho_{L, \max }$ & $\Delta_{L, \min }$ & $\Delta_{L, \max }$ & $\overline{\boldsymbol{E}}(\%)$ \\
\hline 1 & 1 & 1 & 0.4758 & 0.4904 & -0.2386 & 0.2273 & 2.45 \\
3 & 5 & 1 & 0.4979 & 4.2107 & -0.2503 & 0.2027 & 2.44 \\
100 & 5 & 1 & 0.4907 & 0.4994 & -0.2463 & 0.2079 & 2.49 \\
3 & 5000 & 1 & 0.0108 & 1.2329 & -0.0150 & 0.3717 & 2.8 \\
3 & 5 & 1000 & 0.4898 & 0.5005 & -0.24586 & 0.2106 & 2.4 \\
\hline
\end{tabular}

$$
\begin{gathered}
y_{K}=C_{1} x, \\
z_{K}=C_{2} x+D_{21} w_{K}+D_{22}\left(\rho_{K}\right) u_{K} .
\end{gathered}
$$

The iterative LPV control design (25) is performed using the control-oriented model (32) with different $R, D, T$ parameter triads, see Table 1. In the example the impact of the parameter selection is examined. It can be seen that the increase of $R$ results in smaller $\varrho_{L}$ range. Similarly, $D$ reduces the range of $\Lambda$ and the reduction of $\bar{E}$ can be achieved by high $T$ value. Thus, the resulted control strategy can be effectively tuned by the selection of the parameters in (25). An illustrative example on the convergence of the method is found in Figure 3. During the optimization process a trust-region-reflective algorithm ${ }^{23}$ is used, in which each iteration involves the approximate solution using the method of preconditioned conjugate gradients. Figure 3 illustrates that the convergences of the cost function, $\bar{E}$ and each variable are achieved.

\section{2 | Simulation examples}

In the first example the efficiency of the control is examined on a road profile, which is used in the training set of the neural network, see Figure 4A. In the following simulation examples the control strategy with the weights $R=3$, $D=5, T=1$ is selected. Three control strategies are compared in the simulation scenario. First, a LPV control (27) based on the presented NLPV model (26) is designed (NLPV), in which only one scheduling variable $\rho=\Phi(x)$ is used. Second, the system with the actuation of the trained neural network $u=u_{L}$ is examined (NN). Third, the proposed iterative NLPV-based control strategy is designed and analyzed (NLPV-NN). The parameters of the quarter-car model and the magnetorheological damper model are based on the identification of a test-bench with $1 / 5$ scaled real vehicle. $^{24}$

The vertical acceleration signals with each controllers are illustrated in Figure 4B,C. It is shown that the neural network with the prediction of the road profile has good performance in the minimization of the acceleration: the peak values and the oscillation of the signal are reduced. The proposed NLPV-NN control has the same impact on the vertical acceleration. Nevertheless, the compression is only slightly increased, which is shown in Figure 4D,E. The differences in the control interventions are shown in Figure 5A,B. The interventions of NN $\left(u_{L}\right)$ and NLPV-NN $\left(u_{K}\right)$ are close to zero, with which the minimization of the acceleration is prioritized. However, in case of the NLPV control the intervention due to $u_{s t}$ is around 0.5 , which results in a degradation in $\ddot{z}_{s}$. The iteration results in small difference between $u_{K}$ and $u_{L}$. The scheduling variable $\rho_{L}$ and the measured disturbance $\Delta_{L}$ are shown in Figure 5C,D.

In the second example road profile is incorporated in the training set of the neural network. $z_{r}$ has higher peak values and higher frequency components in the excitation, see Figure 6A. It shows that the neural network has less efficiency and the performances are degraded. The vertical acceleration signal of the sprung mass is shown in Figure 6B,C. Although the neural network provides increased acceleration values (see signal NN), the proposed NLPV-NN control strategy guarantees the reduction of the peak values. In case of $\mathrm{NN}$ the degradation in the performance level of $z_{s}-z_{u s}$ is illustrated in Figure 6D,E. The NLPV-NN controller is able to provide the reduction of the compression, even if $u_{L}$ may produce increased compression. Thus, $u_{K}$ can differ more significantly from $u_{L}$, see Figure 7A. It shows that $\rho_{L}, \Delta_{L}$ reach their bounds in $\varrho_{L}, \Lambda_{L}$, see Figure 7B,C.

The third example presents the efficiency of the proposed control structure on a road profile, which contains periodical excitation, see Figure 8A. Since the road profile differs from the samples in the training set of the neural network, 


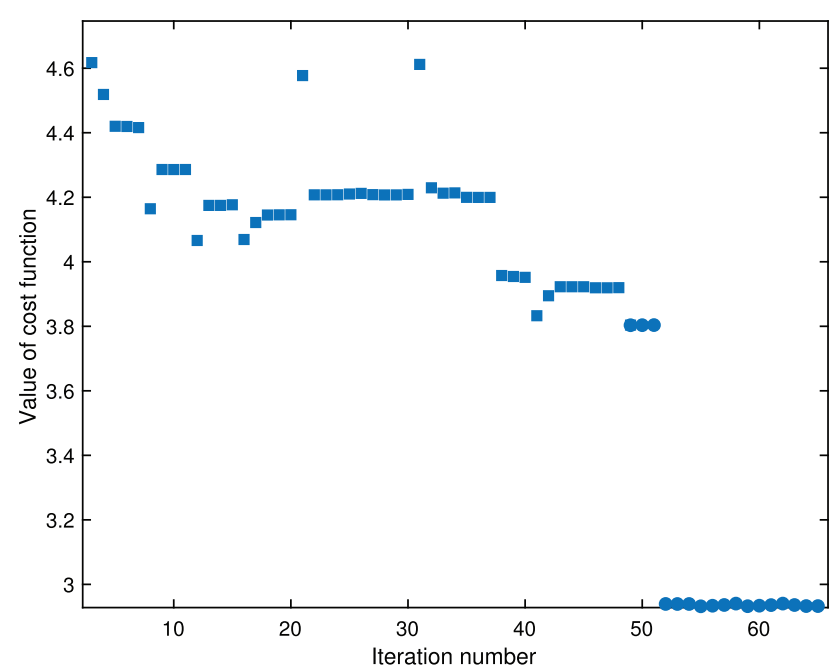

(A) Value of the cost function

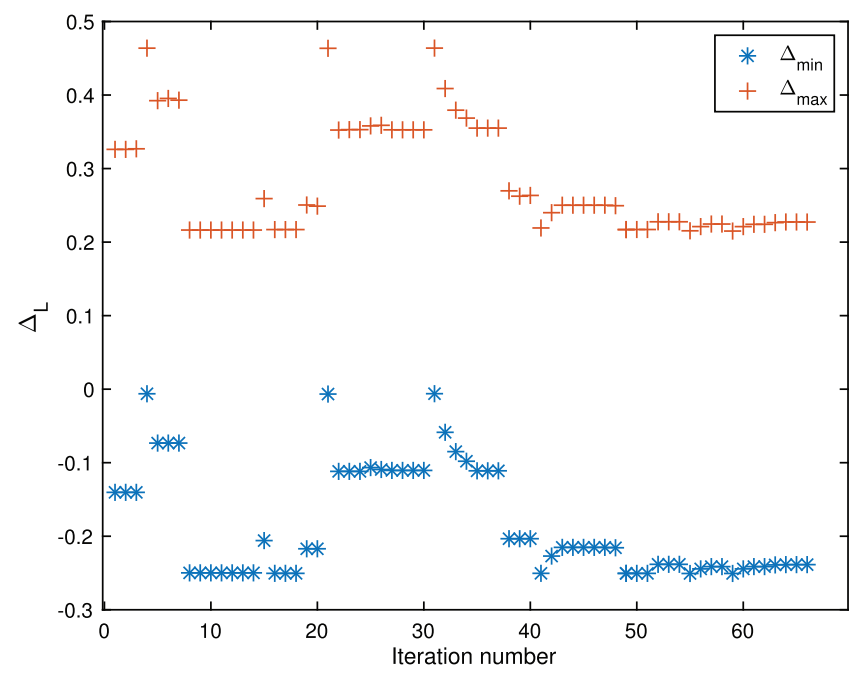

(C) Values of $\Delta_{L, \min }, \Delta_{L, \max }$

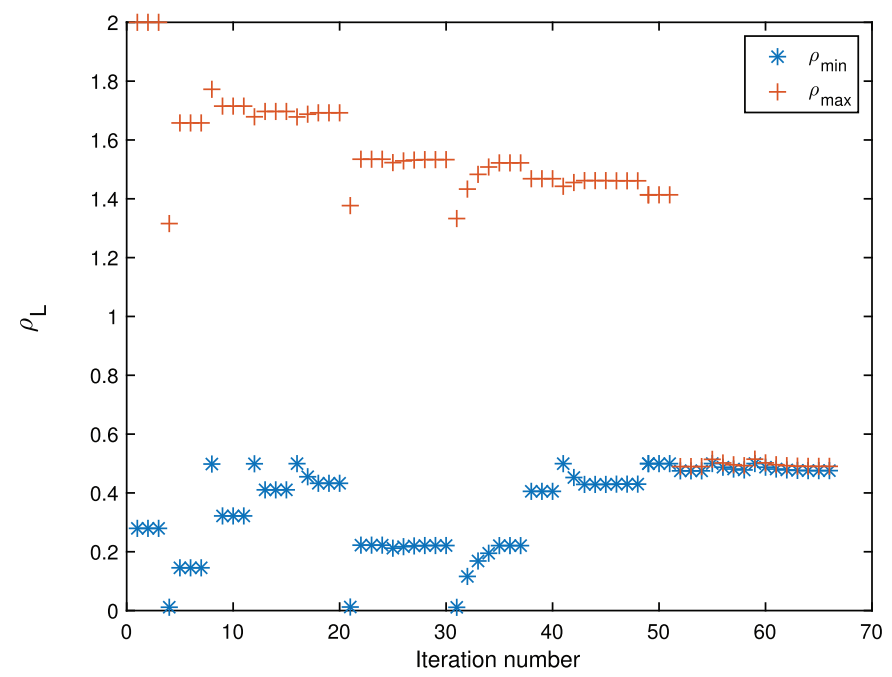

(B) Values of $\rho_{L, \min }, \rho_{L, \max }$

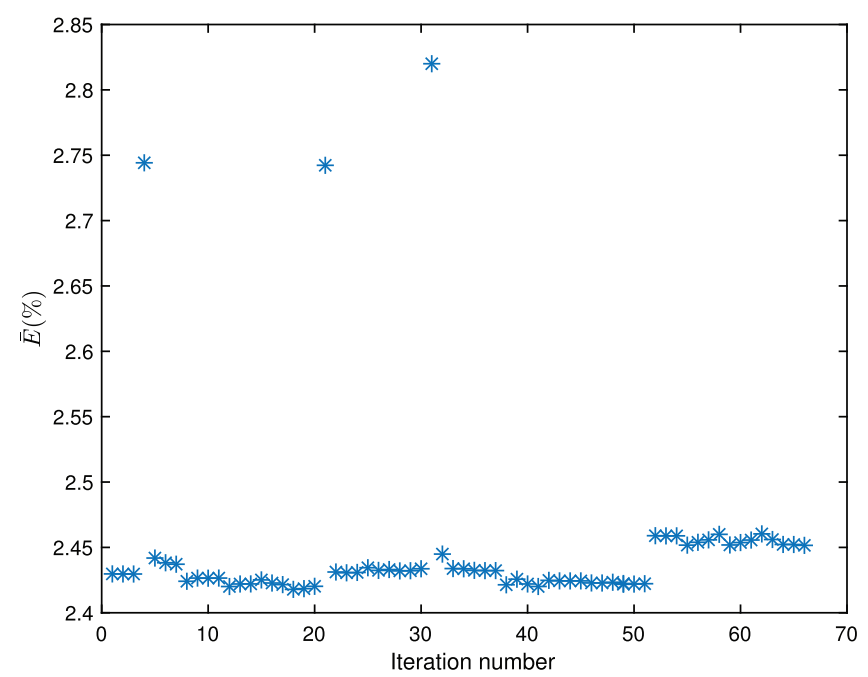

(D) Value of $E$

F I G U R E 3 Illustration on the convergence of the iteration

the application of the neural network as a controller leads to performance degradation. The vertical acceleration and the compression of the suspension have increased values, see Figure $8 \mathrm{C}, \mathrm{D}$. The degradation is limited by the proposed NLPV-NN control strategy, which is achieved by the guaranteed performance level. Thus, $u_{L}$ and $u_{K}$ differ at the final parts of all the periods of the excitation signal, see Figure 8B. It is also shown in the variation of $\rho_{L}$ and $\Delta_{L}$, see Figure 8E,F.

The evaluation of the proposed controller is also carried out through the performance indexes regarding comfort and road holding performances. ${ }^{26}$ The results are illustrated in Figure 9, which contains the numerical values of the resulting performance indexes. The performance indexes are computed through the approximation of the frequency responses regarding the vertical acceleration and the compression signals. The approximation is based on the computation of the power spectral density, see Reference 26 . In the evaluation the passive suspension controller with $u \equiv 0$ is considered to be the basis for the comparison. The performance indexes confirm that the designed NN controller is able to improve acceleration, which is related to comfort. Moreover, the performances of the proposed NLPV-NN controller are very close the NN controller. The contribution of the evaluation is that the proposed guaranteed NLPV-NN controller is able to preserve the maximum performance level of the $\mathrm{NN}$ controller, while it guarantees that the minimum performance level has been provided. 
F I G U R E 4 Simulation example under conventional road profile

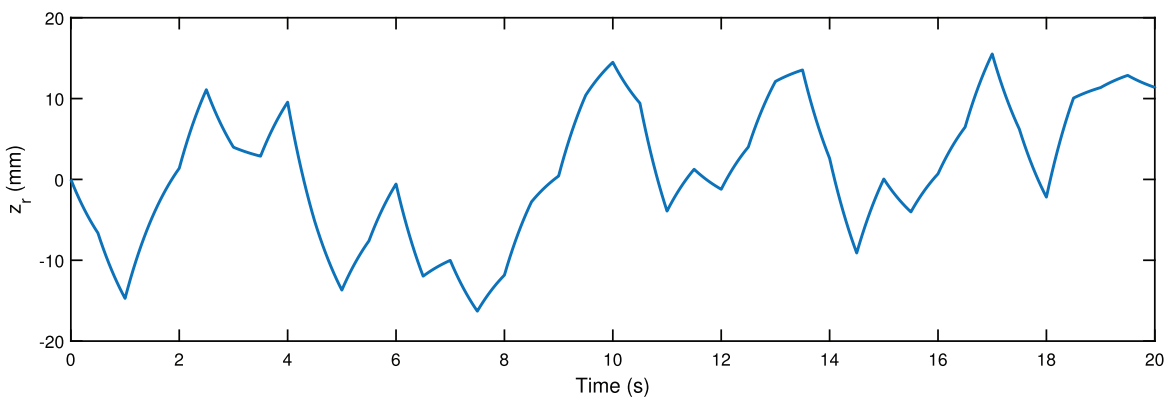

(A) Road profile

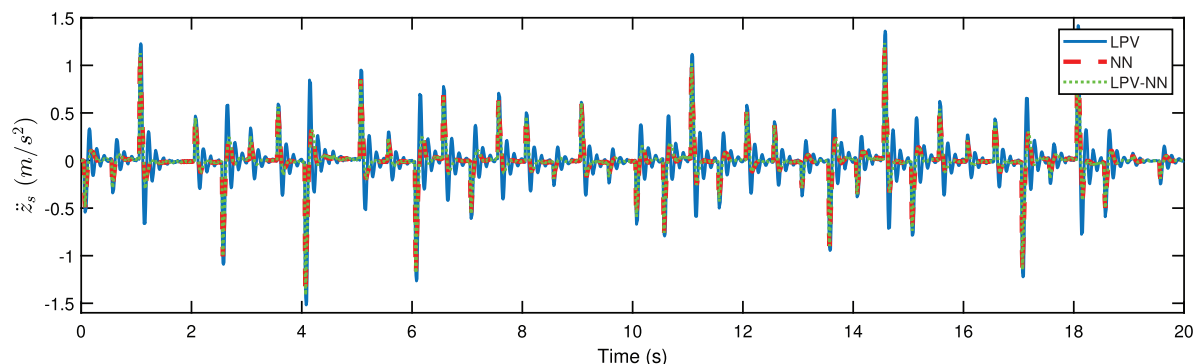

(B) Acceleration

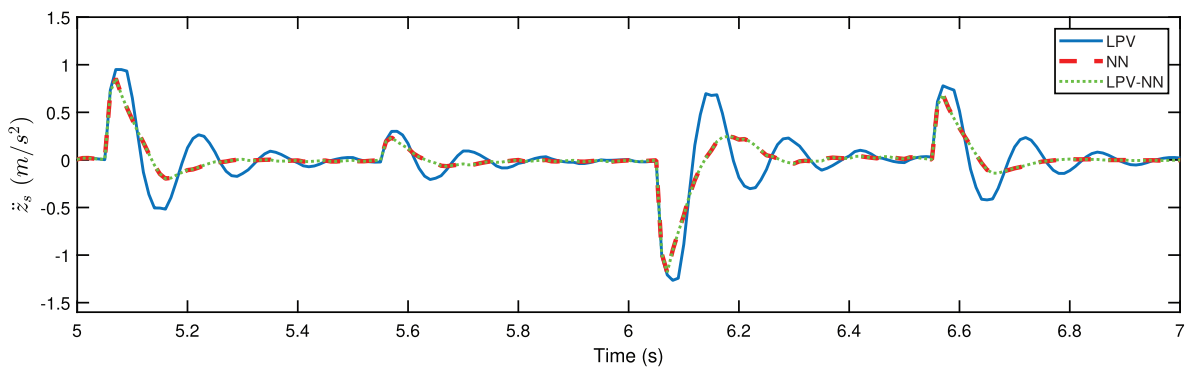

(C) Acceleration between $5 s \ldots 7 s$

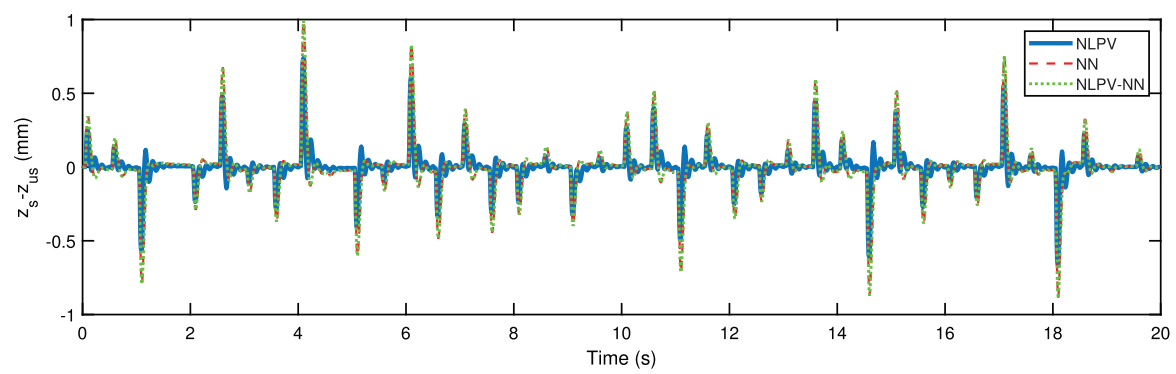

(D) Compression

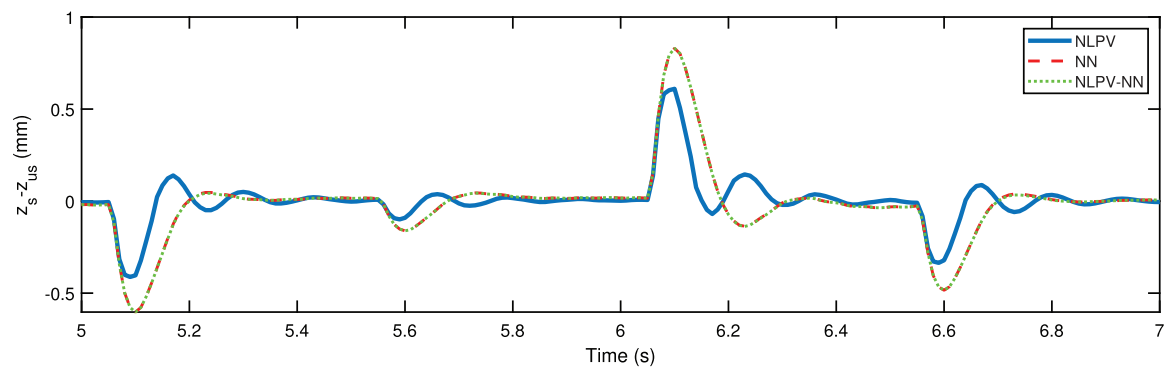

(E) Compression between $5 s \ldots 7 s$ 


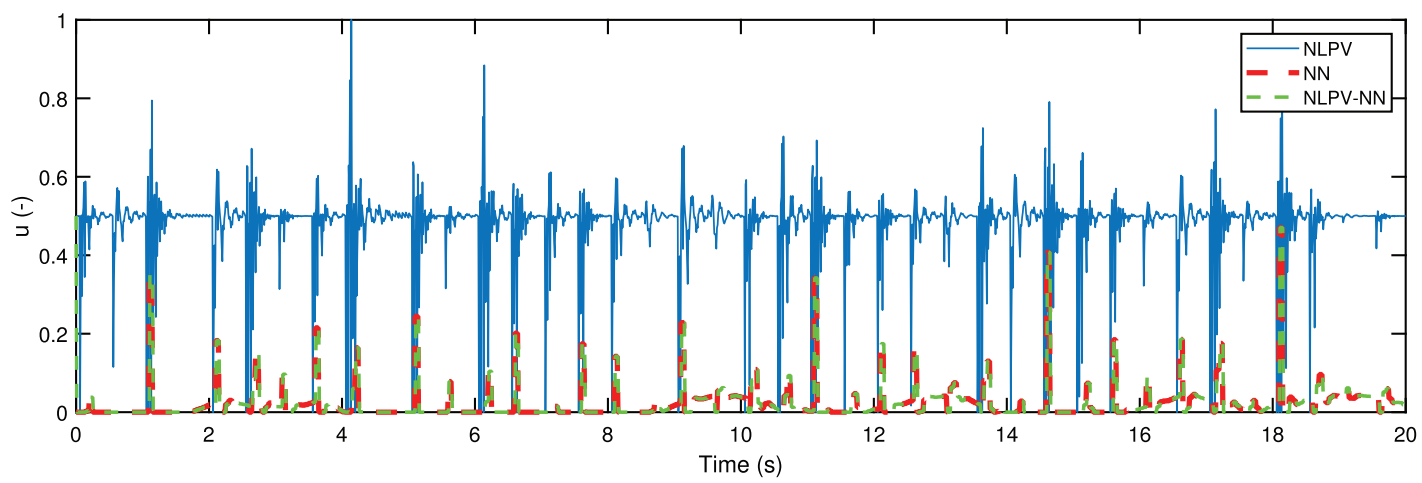

(A) Control intervention

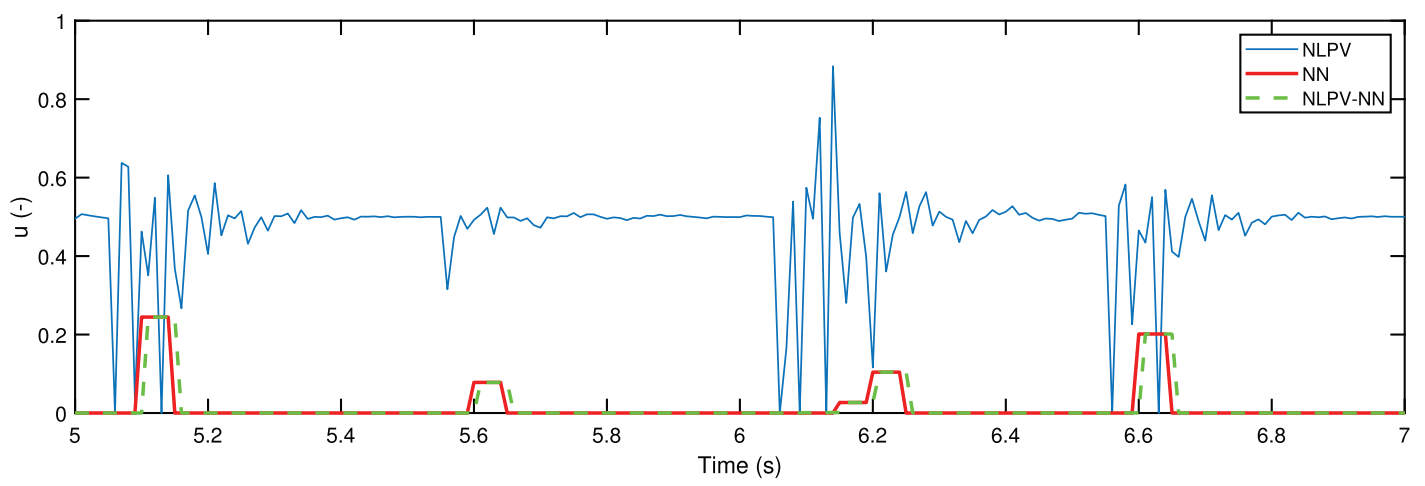

(B) Control intervention between $5 s \ldots 7 s$

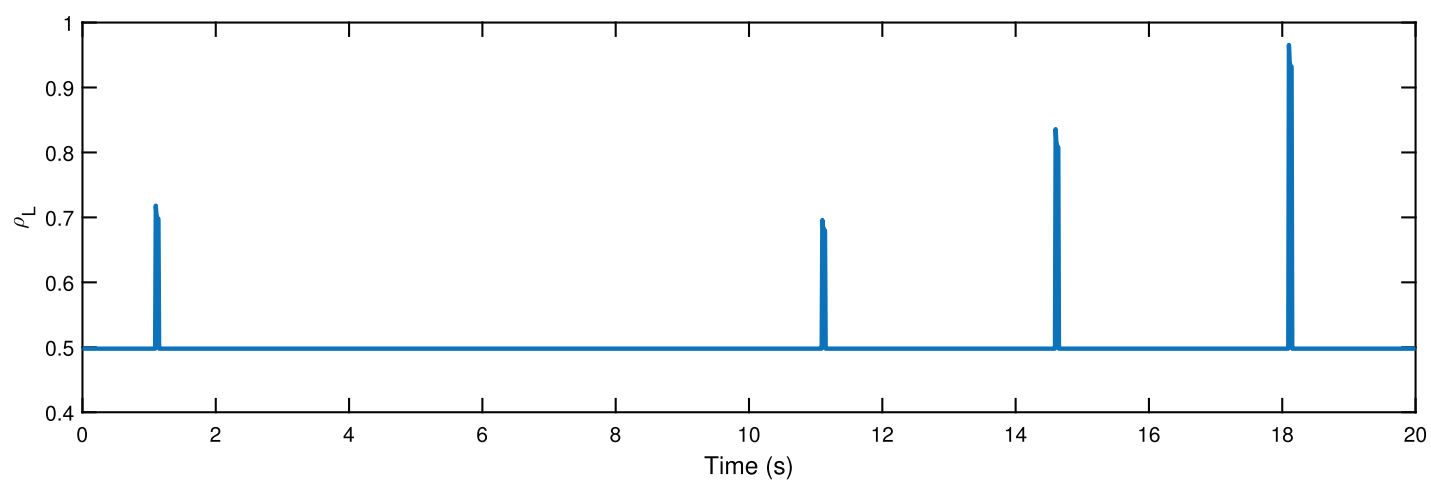

(C) $\rho_{L}$

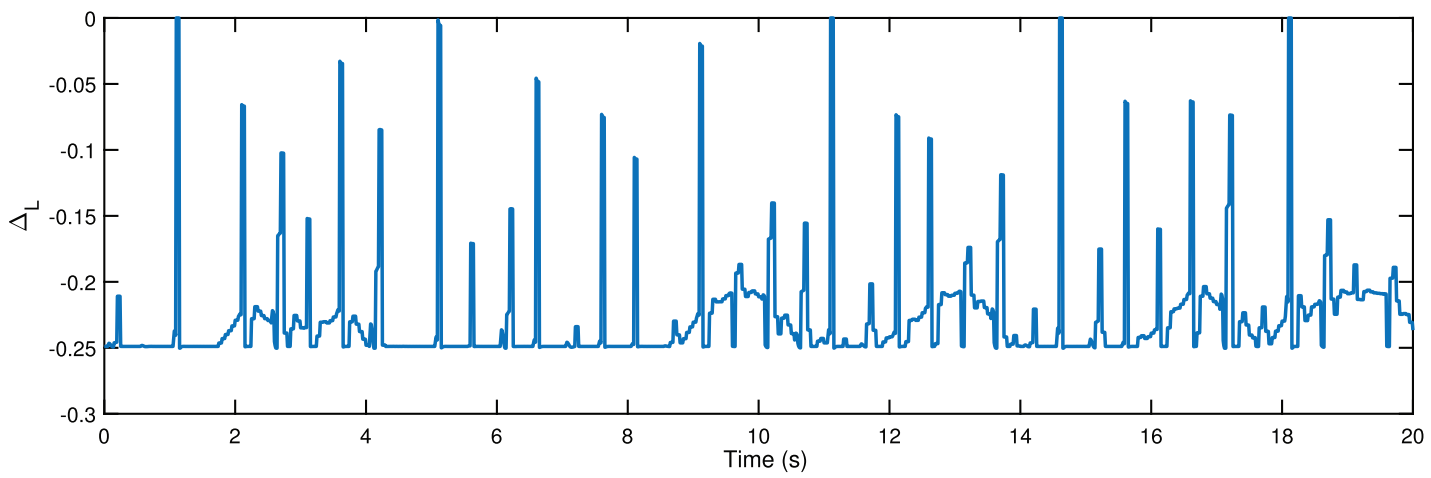

(D) $\Delta_{L}$

F I G U R E 5 Simulation example under conventional road profile (cont.) 


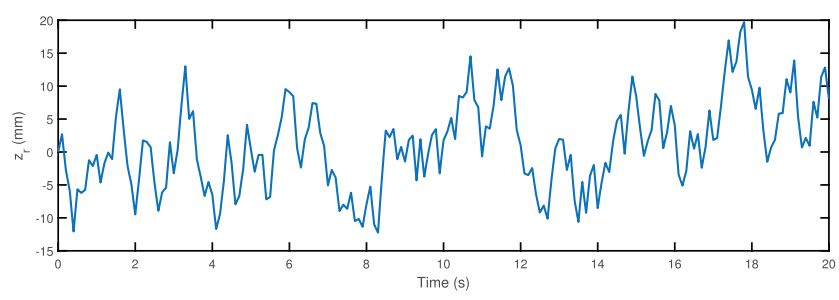

(A) Road profile

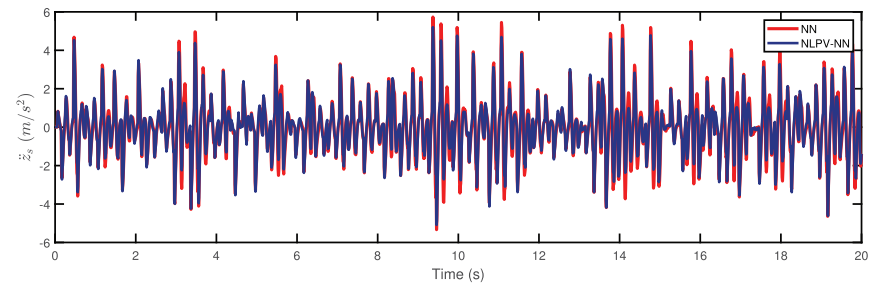

(B) Acceleration

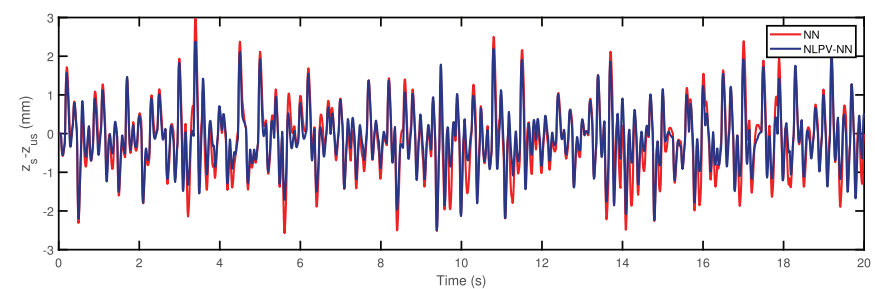

(D) Compression

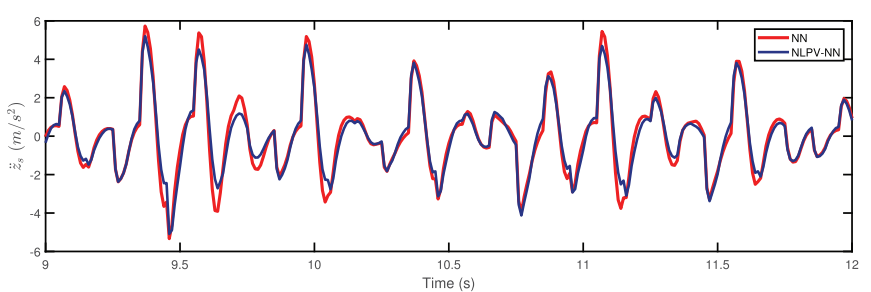

(C) Acceleration between $9 s \ldots 12 s$

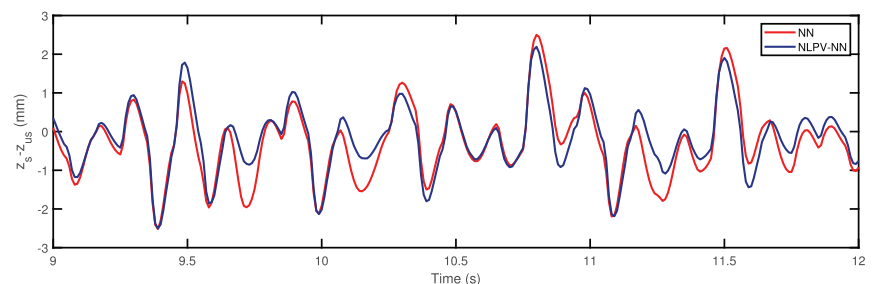

(E) Compression between $9 \quad \ldots 12$

F I G U R E 6 Simulation example with high $z_{r}$ values

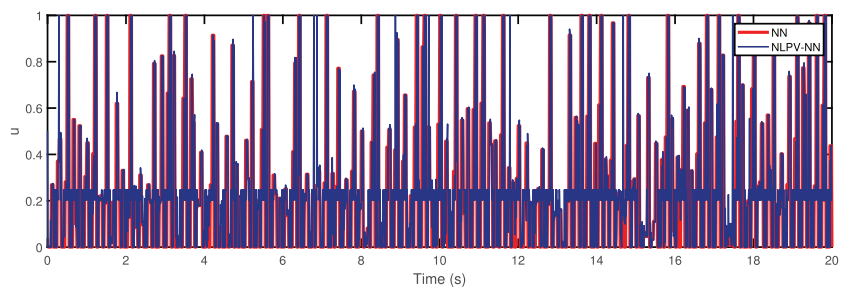

(A) Control interventions

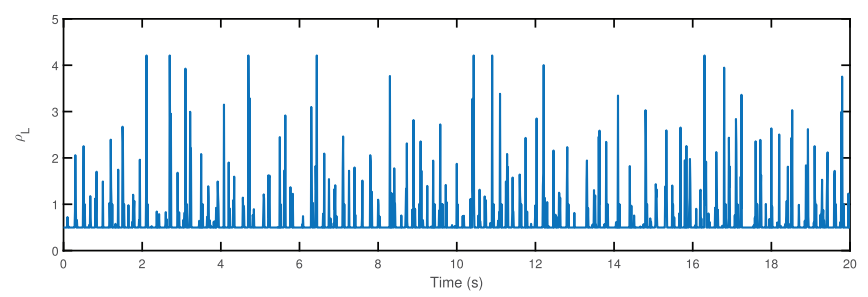

(B) $\rho_{L}$

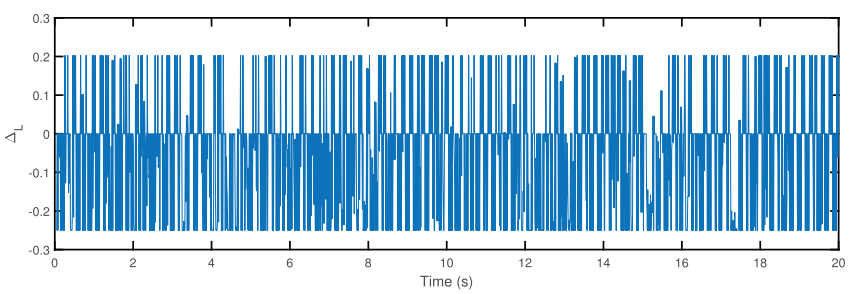

(C) $\Delta_{L}$

F I G U R E 7 Simulation example with high $z_{r}$ values (cont.) 


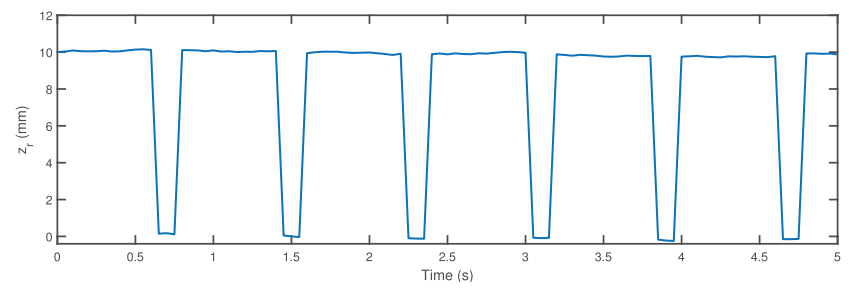

(A) Road profile

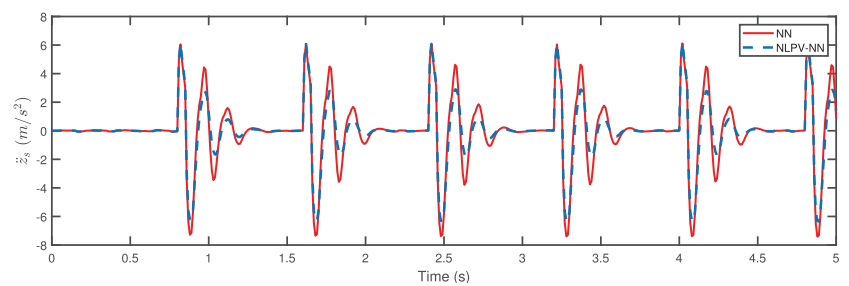

(C) Accelerations

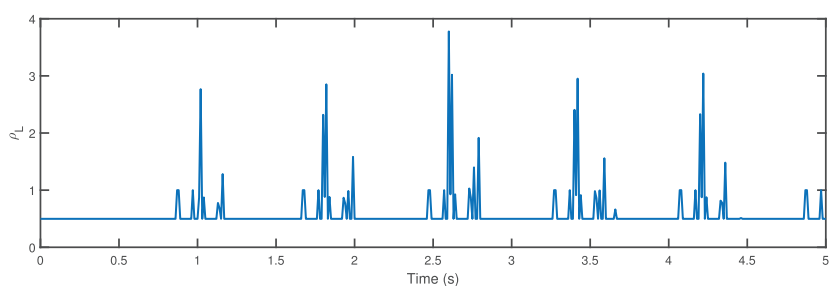

(e) $\rho_{L}$

F I G U R E 8 Simulation example with periodical excitation

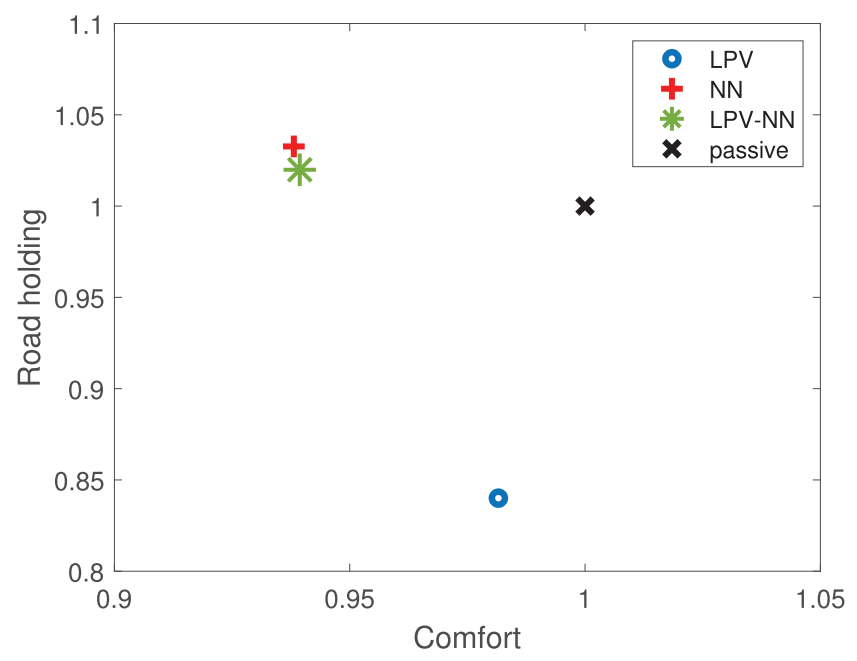

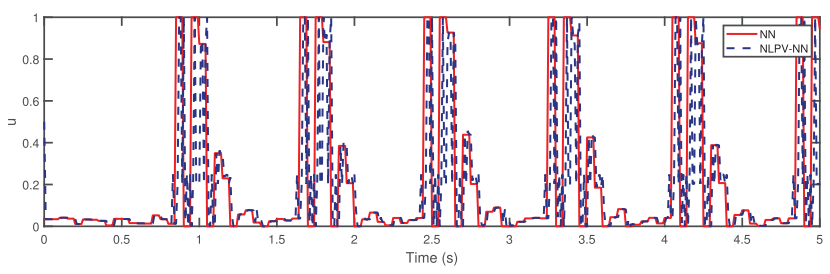

(B) Control interventions

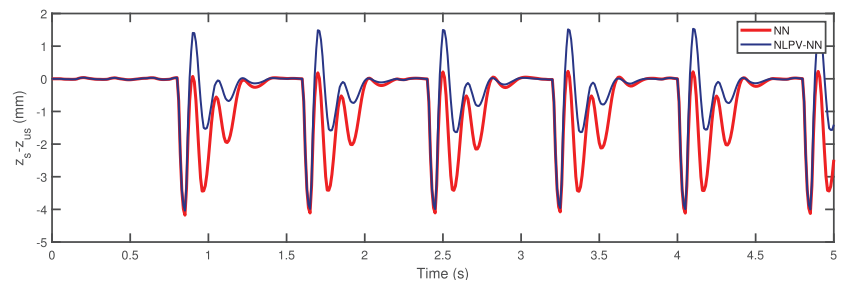

(D) Compressions

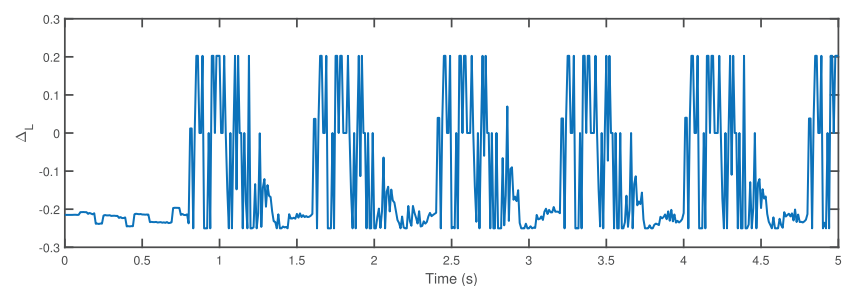

(f) $\Delta_{L}$

F I G U R E 9 Evaluation of the controllers based on performance indexes

\section{6 | CONCLUSIONS}

The article has proposed a method to guarantee the minimum performance level of a control system, which can incorporate in nonconventional controllers, for example, machine-learning-based agents. The LPV-based method achieves its solution in an iterative way. The effectiveness of the method is presented through simulation examples of the control design for semiactive suspension. They are demonstrated that the performance of the system can be preserved through the proposed NLPV-based control design structure. Moreover, the simulation examples illustrate that the resulting control structure can be effectively tuned through the design parameters in the optimization process. 


\section{ACKNOWLEDGEMENTS}

The research was supported by the National Research, Development and Innovation Office through the project "Integration of velocity and suspension control to enhance automated driving comfort in road vehicles" (NKFIH 2018-2.1.13-TÉT-FR). The work of Balázs Németh was partially supported by the János Bolyai Research Scholarship of the Hungarian Academy of Sciences and the ÚNKP-20-5 New National Excellence Program of the Ministry for Innovation and Technology.

\section{ORCID}

Balázs Németh (1) https://orcid.org/0000-0003-0211-3204

\section{REFERENCES}

1. Savaresi SM, Poussot-Vassal C, Spelta C, Sename O, Dugard L. Semi-Active Suspension Control Design for Vehicles. Oxford, UK: Butterworth-Heinemann; 2010.

2. Codevilla F, Muller M, Lopez A, Koltun V, Dosovitskiy A. End-to-end driving via conditional imitation learning. Paper presented at: Proceedings of the 2018 IEEE International Conference on Robotics and Automation (ICRA), Brisbane, QLD, Australia; 2018:4693-4700.

3. Wang Q, Chen L, Tian B, Tian W, Li L, Cao D. End-to-end autonomous driving: an angle branched network approach. IEEE Trans Veh Technol. 2019;68(12):11599-11610.

4. Dossa RFJ, Lian X, Nomoto H, Matsubara T, Uehara K. Human-like agent based on a hybrid of reinforcement and imitation learning. Paper presented at: Proceedings of the 2019 International Joint Conference on Neural Networks (IJCNN), Budapest, Hungary; 2019:1-8.

5. Chen S, Wang M, Song W, Yang Y, Li Y, Fu M. Stabilization Approaches for Reinforcement Learning-Based End-to-End Autonomous Driving. IEEE Trans Veh Technol. 2020;69(5):4740-4750.

6. Zhai L, Chai T, Ge SS. Stable Adaptive Neural Network Control of Nonaffine Nonlinear Discrete-Time Systems and Application. Paper presented as: Proceedings of the 2007 IEEE 22nd International Symposium on Intelligent Control, Singapore, 2007 , pp. 602-607.

7. Zhao D, Chen W, Wu J, Li J. Globally stable adaptive tracking control for uncertain strict-feedback systems based on neural network approximation. Asian J Control. 2016;18(2):527-538.

8. Rosolia U, Borrelli F. Learning model predictive control for iterative tasks. a data-driven control framework. IEEE Trans Autom Control. 2018;63(7):1883-1896.

9. Du Y, Liu C, Li Y. Velocity control strategies to improve automated vehicle driving comfort. IEEE Intell Transp Syst Mag. 2018;10(1):8-18.

10. Göhrle C, Schindler A, Wagner A, Sawodny O. Model predictive control of semi-active and active suspension systems with available road preview. Paper presented at: Proceedings of the 2013 European Control Conference (ECC), Zurich, Switzerland; 2013:1499-1504.

11. Caliskan K, Henze R, Kukukay F. Potential of road preview for suspension control under transient road inputs. IFAC-PapersOnLine. 2016;49(3):117-122.

12. Nguyen MQ, Canale M, Sename O, Dugard L. A model predictive control approach for semi-active suspension control problem of a full car. Paper presented at: Proceedings of the 2016 IEEE 55th Conference on Decision and Control (CDC), Las Vegas, NV; 2016:721-726.

13. Alcantara DH, Morales-Menendez R, Mendoza RAR. Teaching semi-active suspension control using an experimental platform. Paper presented at: Proceedings of the 2016 American Control Conference (ACC), Boston, MA; 2016:7334-7339.

14. Kim S, Kim H, Kang D. Vibration control of a vehicle active suspension system using a DDPG algorithm. Paper presented at: Proceedings of the 2018 18th International Conference on Control, Automation and Systems (ICCAS), Daegwallyeong, South Korea; 2018:1654-1656.

15. Ming L, Yibin L, Xuewen R, Shuaishuai Z, Yanfang Y. Semi-active suspension control based on deep reinforcement learning. IEEE Access. 2020;8:9978-9986.

16. Do AL, Sename O, Dugard L, Savaresi S, Spelta C, Delvecchio D. An extension of mixed sky-hook and add to magneto-rheological dampers. IFAC Proc Vols. 2010;43(21):25-31.

17. Wu F, Yang XH, Packard A, Becker G. Induced $\mathrm{L}_{2}$ norm controller for LPV systems with bounded parameter variation rates. J Robust Nonlinear Control. 1996;6:983-988.

18. Briat C. Stability analysis and control of a class of LPV systems with piecewise constant parameters. Syst Control Lett. 2015;82:10-17.

19. Yang D, Zong G, Karimi HR. Ho refined antidisturbance control of switched LPV systems with application to aero-engine. IEEE Trans Ind Electron. 2020;67(4):3180-3190.

20. Sename O, Gáspár P, Bokor J. Robust Control and Linear Parameter Varying Approaches. Berlin, Germany: Springer Verlag; 2013.

21. Gáspár P, Szabó Z, Bokor J, Németh B. Robust Control Design for Active Driver Assistance Systems. A Linear-Parameter-Varying Approach. New York, NY: Springer Verlag; 2017.

22. Lagarias JC, Reeds JA, Wright MH, Wright PE. Convergence properties of the nelder-mead simplex method in low dimensions. SIAM J Optim. 1998;9(1):112-147.

23. Coleman TF, Li Y. An interior, trust region approach for nonlinear minimization subject to bounds. SIAM J Optim. 1996;6:418-445. 
24. Pham T-P, Sename O, Dugard L. Real-time damper force estimation of vehicle electrorheological suspension: a nonlinear parameter varying approach. IFAC-PapersOnLine. 2019;52(28):94-99.

25. Guo S, Yang S, Pan C. Dynamic modeling of magnetorheological damper behaviors. J Intell Mater Syst Struct. 2006;17(1):3-14.

26. Poussot-Vassal C, Savaresi SM, Spelta C, Sename O, Dugard L. A methodology for optimal semi-active suspension systems performance evaluation. Paper presented at: Proceedings of the 49th IEEE Conference on Decision and Control (CDC), Atlanta, GA, $2010 ; 2892-2897$.

How to cite this article: Németh B, Gáspár P. Ensuring performance requirements for semiactive suspension with nonconventional control systems via robust linear parameter varying framework. Int J Robust Nonlinear Control. 2021;31:8165-8182. https://doi.org/10.1002/rnc.5282 